\title{
A model to calculate electrostatic charge structure of eruptive clouds from volcanic eruptions
}

\author{
Vladimir Uvarov ${ }^{1}$, Rinat Akbashev ${ }^{2}$, Pavel Firstov ${ }^{1,2}$ and Nina Cherneva ${ }^{1, \star}$ \\ ${ }^{1}$ Institute of Cosmophysical Research and Radio Wave Propagation FEB RAS, Kamchatskiy kray, Paratunka, \\ Russia \\ ${ }^{2}$ Kamchatka Branch of FRC GS RAS, Petropavlovsk-Kamchatksiy, Russia
}

\begin{abstract}
The paper proposes a model to calculate electrostatic charge structure of an eruptive cloud (EC) based on the response of atmospheric electric field (AEF $E_{Z}$ ) strength during cloud passage near an observation site. The model provides high identity of calculated and experimental data for complex configurations of EC electrostatic charges. Examples of calculation of EC structures from Shiveluch volcano (Kamchatka) eruptions are given. They were estimated with the help of the developed model based on the records of AEF $E_{Z}$ response at the distances of 45 and $113 \mathrm{~km}$.
\end{abstract}

Key words: volcano, eruption, eruptive cloud, electrostatic charge

\section{Introduction}

Generation of atmospheric electrostatic field (AEF) is associated with multiple processes of planetary and local scales. Global electric circuit is determined by global lightning sources [1]. Moreover, at the local level, AEF is connected with such local parameters, affecting electrization mechanism, as conductivity, temperature, viscosity, electron and ion concentration, ionization source strength, aerosol concentration and content and others [2]. Cloud electrization of meteorological origin primarily refers to local sources of AEF variations [3].

For the Kamchatka peninsula and a number of other regions of the globe where volcanic activity manifests itself, EC electrization is an intensive additional factor. The electrization occurs as the result of volcanic eruption $[4,5]$. The main difference between the clouds of meteorological origin and ECs is that ECs, initiated by volcanic explosive activity, are loaded with significant volume of fine grained ash and aerosols and have high temperature contrast at cloud-atmosphere boundary $[6,7]$. Moreover, depending on the character of explosive activity, volcanic ash content, amount of water dissolved in magma and so on, EC electrization is conceivable within wide limits of charge value and with different polarization [8-13]. These circumstances as well as the interaction of EC boundary with the clouds of meteorological origin cause lightning activity. Lightning stroke location by modern techniques allows one to trace EC propagation $[14,15]$.

\footnotetext{
${ }^{\star}$ Corresponding author: nina@ikir.ru

The paper was carried out within the framework on the subject «Dynamics of physical processes in active zones of near space and geospheres» (AAAA-A17-117080110043-4) and supported by RFBR Grant 19-05-00543 (AAAA-A19-119041790002-0).
} 
EC evolves during its propagation: fractionation during weighted component sedimentation, aerosol formation, its temperature decrease $[16,17]$ that is reflected in EC electrostatic structure $[12,13]$. Near-field electrostatic processes in EC are indirect signs of eruption character [18] whereas in the far-field zone, the conditions of EC propagation under wind effect make significant impact on its electrization.

In the course of investigation of EC electric structure, observations of atmospheric electric field vertical component $\left(\mathrm{AEF} E_{Z}\right.$ ) by ground-based electrostatic fluxmeters were very popular [7, 13, 19, 20]. When interpreting the obtained data and calculating EC charge, one of the following models is usually applied: field of one effective charge, field of uniformly charged ellipsoid body [21] or dipole field [5]. Such an electric structure of a cumulonimbus cloud is represented by at least 4 main space charges, two main ones of which form a vertical dipole [22]. Simulation of a field of differently originated clouds is quite illustrative and thus is widely used for qualitative description. Field simulation by uniformly charged ellipsoid is used, as a rule, for massive cloud formations [23]. In order to describe such a model, 6 parameters (3 diameters and 3 orientation angles) are required, but in most cases it is difficult to calculate them.

EC formed under wind effect are usually characterized by small vertical dimensions, significant extent along the drift direction and small crosswise dimensions. As opposed to the vertical electric structure of meteorological clouds, horizontal structure is typical for EC. In this case, to describe field sources, we need to apply several ellipsoids of different polarity and orientation that makes the model more complicated and hardly suitable for quantitative analysis.

When describing a symmetric dipole, one needs 7 components: 6 coordinates of charge position and one for charge value. However, in the majority of cases, real aero-electric structures are asymmetric. Thus, it is necessary to add a unipolar charge to the dipole for the approximation to be satisfactory. As a result we have that in a simplest case we need to obtain 8 unknown quantities from experimental values for quantitative application of such a model. Applying such a model, EC electric structure with significant horizontal dimensions should be described by several dipoles. That complicates model application and makes it problematic to use it for EC electric structure investigation.

\section{Volcanic globule}

To construct a model of a charged EC, we refer to the known notions in electrostatics. The basis of them is detection of a uniformly charged volume element the field of which can be approximated by point charge field. Combination of such fields into more complicated configurations (dipoles, quadruples etc.) allows one to approximate a field of arbitrary configuration with required accuracy. As the simplest element of EC electrostatic structure we take a compact uniformly-charged space of diameter $D c$ and charge $Q$ which is called «volcanic globule» (VG). As a rule, the observed typical VG dimensions are significantly less than the distance from a source to the observation site. Thus, we approximate VG field by point charge field with sufficient accuracy. For the VG field with significant horizontal dimensions we introduce an additional parameter taking into account its extention.

Such an approach allows us to turn to the model approximating EC electric field by the superposition of fields of separate electrostatic structures of simplest forms, VG. Under certain assumptions, BG parameters are determined from the data obtained by ground-based electrostatic fluxmeters. Thus, description of EC electrostatic structure is reduced to the superposition of VG set charges fields. Volcanic activity is reflected indirectly in EC, thus, EC electrostatic field decomposition on the basis of partial fields of separate VG may give an idea on volcanic activity character if we take into account the evolution processes occurring as the result of its motion along the transfer direction under wind effect. 


\section{Description of the model}

A mathematical model always contains some limitations since it represents a certain degree of idealization of a real situation. In this case, to construct our model, we have to assume that relaxation processes are absent. It is fair if the characteristic time of relaxation evolution of EC separate components is more than that of their position change.

We consider rectangular coordinates with the origin at the point of sensor location with the vertical axis $Z$ and horizontal axis $X$, oriented along VG motion, and axis $Y$ perpendicular to it (Fig.1). Dynamics of VG motion in such a coordinate system is determined by wind velocity $v$ at the height $z$ and time $t-t_{\text {niar }}$ is the moment of the closest distance to VG, $x=v \cdot\left(t_{i}-t_{\text {niar }}\right)$.

When making such a choice of coordinates, VG trajectory projection on the ground surface is at the distance $y$ from the axis $X$. VG center position is determined by the vector $R=n \cdot \sqrt{x^{2}+y^{2}+z^{2}}$, where $n$ is a unit vector directed from the origin of coordinates to VG with the coordinates $x, y, z$. Extreme value of field intensity module $E_{\max }$ is achieved at the distance $R_{\min }=\sqrt{y^{2}+z^{2}}$.

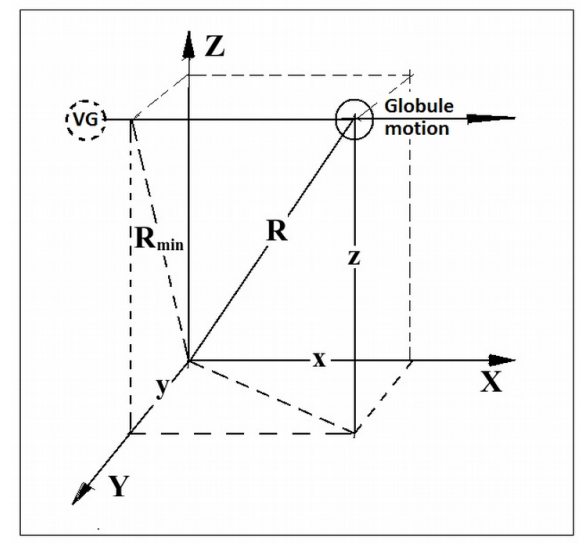

Figure 1. Volcanic globule motion in the model coordinate system

Air conductivity is many orders less than that of the ground. Thus, when constructing the model, we assume that ground conductivity is infinite. Then, VG charge field over the Earth surface is described by the superposition of fields of two equal charges of opposite signs located at equal distances over and under the ground surface and being direct reflections of each other. As a result, the field at the Earth surface level has only a vertical component and can be described by the expression:

$$
E_{z}=A \cdot \Psi
$$

In this expression the first factor which is determined by charge value and the minimum distance to a VG is the field amplitude independent of time and VG location.

$$
A=\frac{Q_{z}}{2 \pi \varepsilon_{0} \cdot R_{\min }^{3}},
$$

where $R_{\min }$ is the minimum distance from a detector to the horizontal projection of VG trajectory, $\varepsilon_{0}$ is the dielectric constant. 
The second factor describes the characteristics of VG field with a unit charge moving under wind effect at the height $z$ along a straight line parallel to axis $X$ with velocity $v$.

$$
\Psi_{i}=\frac{E_{i}}{E_{\max }}=\left\{1+\left(\frac{v\left(t_{i}-t_{\text {extr }}\right)}{\delta}\right)^{2}\right\}^{-3 / 2},
$$

where $t_{i}, t_{\text {extr }}$ is the current time and the time of VG position at the minimum distance from an observation site, $\delta$ is the extension parameter defining VG effective size and controlling curve $\Psi_{i}$ form (Fig.2) under the condition that VG motion corresponds to Fig.1.

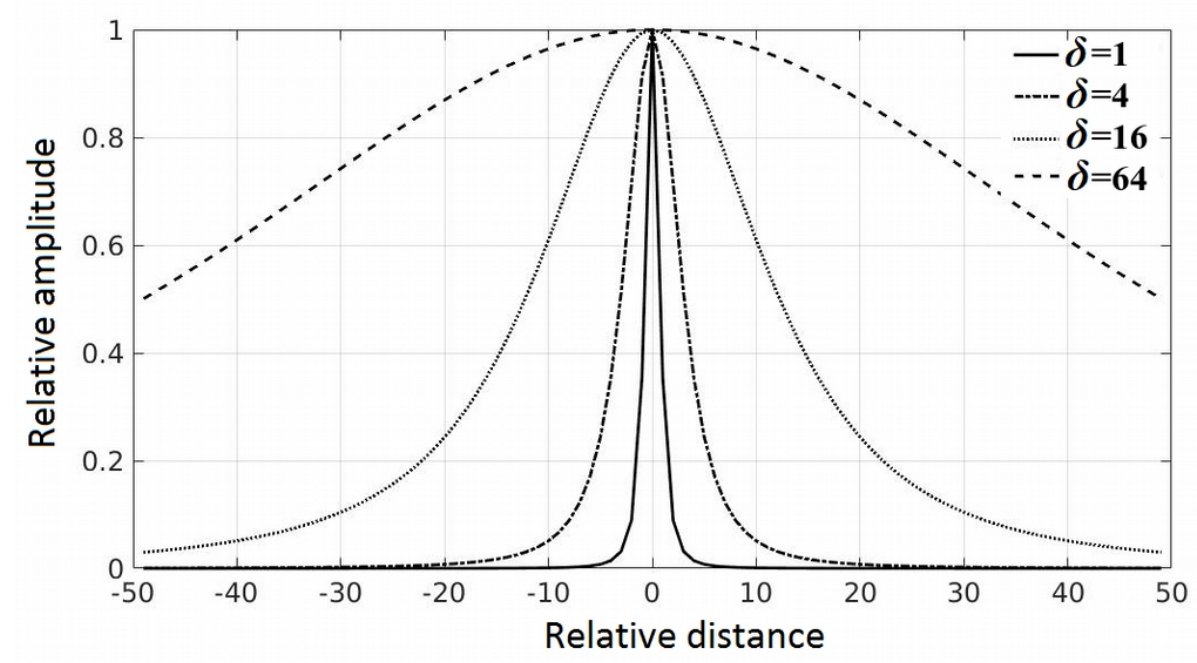

Figure 2. Extension function curves for different parameter $\delta$ values

From the obtained ratios $(1-3)$ we can make the following conclusions:

- VG field is described by an even function becoming a unit at the extremum point and going to zero at infinity;

- at the extremum point, field value is equal to $A$;

- when the height $z$ is known and the distance from a sensor to the horizontal projection of trajectory $y$ is the shortest, applying experimental data we can find VG charge according to (2):

$$
Q=A \frac{2 \pi \varepsilon_{0} \cdot R_{\min }^{3}}{z},
$$

Charge sign is determined by $A$ position relatively the field average value and the width of the curve $\Psi$, describing field structure, is determined by effective extension parameter $\delta_{\text {eff }}$.

\section{VG extension parameter}

As the VG charge from ratio (4) is determined we can determine VG characteristic dimension applying expression (3) by searching for an optimal approximation $M=A \Psi\left(\delta_{o p t}\right)$ of $E_{z}$ data sampling that is formally reduced to optimization problem of the objective function $\frac{\partial}{\partial \delta} \cdot \Phi(\delta)=0$ with respect to the 
control parameter $\delta_{e f f}$, effective parameter of $\mathrm{VG}$ extension. In a general case, this equation does not have an analytical solution and is a typical problem of linear programming.

When choosing the measure of deviation of Euclidean distance between realization and approximating function, the objective function takes the form

$$
\Phi(\delta)=\sum_{i=1}^{N}\left(F_{i}-M_{i}(\delta)\right)^{2},
$$

If $M_{i}=0$, the solution $\frac{\partial}{\partial \delta} \cdot \Phi(\delta)=0$ yields a well-known least-square method. We applied a numerical approach of search for the parameter $\delta_{o p t}$. It was determined by the minimum value of the objective function $\Phi\left(\delta_{o p t}\right)$ within the interval of possible values of the parameter $\delta_{\text {opt }}$. For this purpose, we used a widely used application program package MATLAB for technical and scientific calculations. The package has Optimization Toolbox function oriented on different variants of solution of optimization problems.

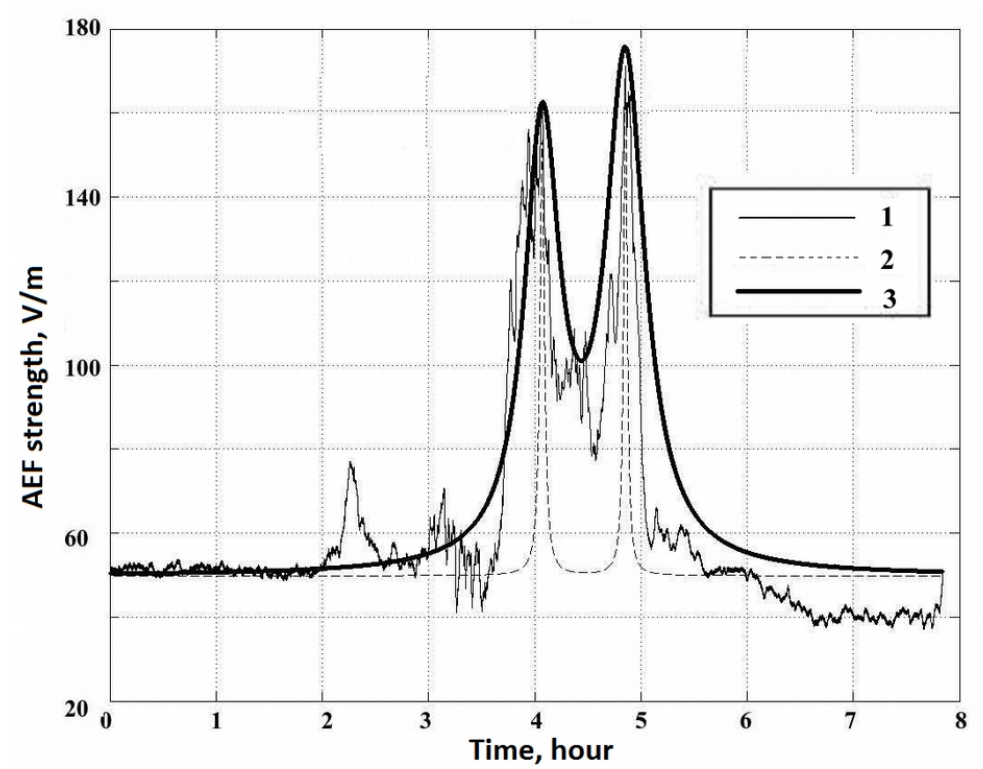

Figure 3. Response in AEF $E_{Z}$ during eruptive cloud passage from the explosive eruption of Shiveluch volcano on November 16, 2014 [23] compared to the calculated curves. 1 - experimental curve; 2 - approximation by the field of two VG point charges; 3 - approximation by the field of two VG optimizing the extension parameter $\delta_{\text {opt }}=1$.

As an example, we consider an explosive eruption which occurred on Shiveluch volcano at 10:17 on November 16, 2014. EC from this eruption rose up to the height of $13 \mathrm{~km}$ above the sea level. Its propagation was observed on the images of Satellite Landsat 8. About two hours later, a positive anomaly of one and a half hour long was observed in AEF $E_{z}$ records at KZYG (Kozyrevsk site) $113 \mathrm{~km}$ to the south-west from Shiveluch volcano [24]. The anomaly looked like a double-peak baylike disturbance with the exceedance $E_{z}=125 \mathrm{~V} / \mathrm{m}$ with respect to the background (Fig.3). According to the satellite data, the time of anomaly occurrence corresponds to that of EC passage $20 \mathrm{~km}$ to the east of KZYG. According to the of maximums propagation times, estimated as differences of times between the beginning of the eruption and their arrivals, we calculated the velocities of motion for 
two parts of the eruptive cloud as $17.7 \mathrm{~m} / \mathrm{s}$ and $10.9 \mathrm{~m} / \mathrm{s}$, respectively. Agreement of aero-electric structure propagation velocities with wind velocities (based on upper-air sounding data) at definite heights indicates the fact that ash propagated at two horizons $(9 \div 10$ and $12 \mathrm{~km})$ where temperature inversions were observed according to upper-air sounding data.

This anomaly was approximated by the field of two VGs with point charges (curve 2, Fig.3). Then the extension parameter $\delta_{\text {opt }}=1$ was optimized (curve 3, Fig.3). The latest case shows significantly better approximation to the experimental curve.

\section{Field decomposition by several globules}

In most of any cases, EC electrostatic field consists of several VGs which appear as the result of each act of magma fragmentation in volcanic conduit. Owing to the strength of applicability of the superposition principle in this case, the resultant field $E_{z}^{\sum}$ can be written as a sum of partial fields of $\mathrm{VG}$ each of which may have its own parameters, charge sign and value as well as extension

$$
E_{z}^{\sum}=\sum_{k} A_{k} \Psi\left(\delta_{k}\right)
$$

The well-known law, connecting the characteristics of a separate source and the structure of the field generated by it $(1-3)$, on the basis of field character allows us to estimate charge values and VG extension parameters responsible for recorded data formation. The most obvious algorithm of decomposition of recorded data by dominant VG fields is the funnel algorithm for approximating field generated by the dominant VG followed by difference series analysis.

Fig.4 illustrates VG partial field decomposition of the vertical electric field for EC from Shiveluch volcano eruption on November 16, 2014. The decomposition was estimated to the level of independence of a root-mean-square deviation relative value on iteration number. The approximation accuracy, applying the described technique, turned to be high enough. Approximation differences (dashed line) from the measurement data (solid line) are obvious only on the right part of Fig.4.

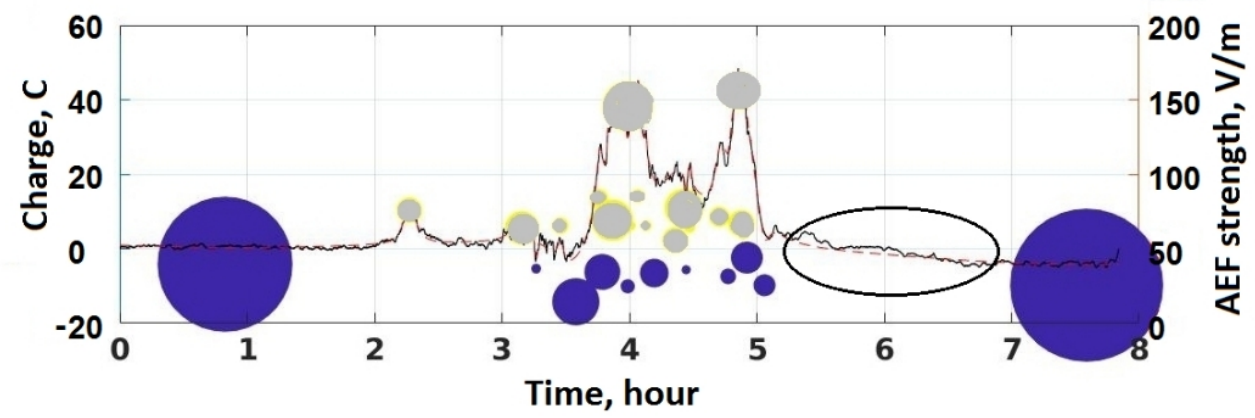

Figure 4. Decomposition of the vertical electric field attributed to Shiveluch volcano eruption on November 16 , 2014 with respect to VG partial fields. Circle area size is proportional to VG effective size. Gray color is positive charge, blue color is negative charge. For the background values relatively $E_{\max }$, the ellipse shows the fragment where the difference of experimental (solid line) and calculated (dashed line) data is obvious

We consider some details of application of the suggested method on the example of AEF Ez experimental data obtained during the passage of the EC from Shiveluch volcano eruption on June 
14, 2017 over the KLYG site (Klyuchi) [25]. In this case, ashfall $\left(100 \mathrm{~g} / \mathrm{m}^{3}\right)$ was observed at the observation site located $45 \mathrm{~km}$ to the north from Shiveluch volcano during the passage of the EC propagating with the velocity of $4-7 \mathrm{~km} / \mathrm{s}$ at the height of $12 \mathrm{~km}$ above the sea level. According to the paper [5], the form of a high-amplitude response in AEF $E_{Z}\left(-6<E_{Z}<5 \mathrm{kV} / \mathrm{m}\right)$ indicated that EC electrostatic structure had the form of a horizontal dipole. We consider application of the method for the case described above on different-scale time series. On 21-hour time segment, the method allowed us to make only large-scale decomposition, detection of a few high-amplitude GV of large effective size (Fig.5a). When the time interval was reduced to anomaly duration, more detailed decomposition on the basis of smaller VG (Fig.5b) was realized.

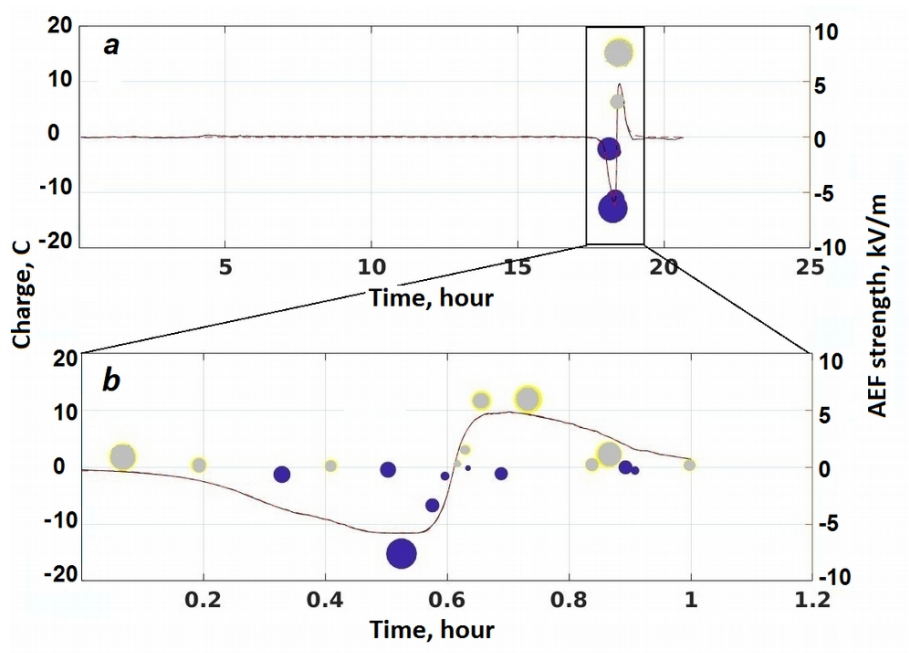

Figure 5. Electric field vertical component during the Shiveluch volcano eruption on June 14, 2017. Decomposition on the sampling with different degree of detail. Symbols are identical to those in Fig.4 a - 21-hour sampling; $\mathrm{b}-1$-hour sampling attributed to EC passage time.

\section{Conclusions}

1. We proposed a model to estimate the eruptive cloud electrostatic charge structure based on the response of atmospheric electric field strength during its passage near an observation site. The model provides high identity of calculated and experimental data for complicated enough configurations of EC electrostatic charges.

2. The basis of the model is the assumption on VG structure stationarity. It is fair for VG having passed the initial stage of formation on time intervals which are shorter than the times of VG measurement.

3. The proposed model allows us to calculate EC electrostatic field of any complexity and provides high quality of experimental data approximation.

The authors appreciate Nagorskiy P.M. for useful criticism and remarks that promoted better information presentation in the paper. 


\section{References}

[1] E.A. Mareev, Physics-Uspekhi, 53(5), 504-511 (2010)

[2] E.R. Williams, "The global electrical circuit: A review", Atmospheric Research, 91, 140-152 (2009). https://doi:10.1016/j.atmosres.2008.05.018

[3] N.V. Krasnogorskaya, Elektrichestvo nizhnikh sloev atmosfery i metody ego izmereniya, (Leningrad, 1972) (in Russian)

[4] N.V. Cherneva, P.P. Firstov, Formirovanie lokal'nogo elektricheskogo polya atmosfery na Kamchatke pod vliyaniem prirodnykh protsessov [Formation of atmospheric local electric field in Kamchatka under natural process effect], (Vladivostok: Dal'nauka, 2018) (in Russian)

[5] N.V. Cherneva, E.A. Ponomarev, P.P. Firstov, A.V. Buzevich, Bulletin of KRAESC. Earth Sciences., 2:10, 60-64 (2007) (in Russian)

[6] K. Aizawa, C. Cimarelli, M.A. Alatorre-Ibarguengoita, A. Yokoo, D. Dingwell, M. Iguchi, Earth and Planetary Science Letters, 444, 45-55 (2016).

[7] S.J. Lane, M.R. James, J.S. Gilbert, Journal of Physics, Conference Series, 301(012004), 1-4 (2011)

[8] H. Hatakeyama, J. Meteor. Soc. Jpn., 27, 372-376 (1949) (in Japanese)

[9] H. Hatakeyama, K. Uchikawa, J. Meteor. Soc. Jpn., 21, 84-89 (1951) (in Japanese)

[10] S.J. Lane, J.S. Gilbert, Japan Bull., 54, 590-594 (1992)

[11] A.W. Woods, Bull Volcanol., 50, 169-193 (1988)

[12] M.R. James, L. Wilson, S.J. Lane, J.S. Gilbert, T.A. Mather, R.G. Harrison, R.S. Martin, Space Sci. Rev., 137, 399-418 (2008) DOI: 10.1007/s11214-008-9362-z

[13] T.A. Mather, R.G. Harrison, R.S. Martin, ServGeophys, 37, 387-432 (2006) DOI: 10/1007/s10712-006-9007-2

[14] S.A. Behnke, S.R. McNutt, Bull Volcanol., 76(847), 2-12 (2014) DOI: 10.1007/s00445-0140847-1

[15] A.R. Van Eaton, À. Amigo, D. Bertin et al., Geophys Res. Letters, 3563-3570 (2016) DOI: 10.1002/2016GL068076

[16] R. Wall, J. Flottau, Aviation Week and Space Technology, 172, 23-25 (2010)

[17] A.R. Van Eaton, M. Herzog, C.J.N. Wilson, J Geophys Res. Sol Ea, 117:B03203 (2012) https://doi:10.1029/2011JB008892

[18] M.R.James, S.J. Lane, J.S. Gilbert, J. Geol. Soc. London, 155, 587-590 (1998)

[19] M. Laruna, The Space Congress, Proceedings, 22-32 (1987)

[20] T. Miura, T. Koyaguchi, Y. Tanaka, Bull Volcanol, 64, 75-93 (2002)

[21] O.D. Kellog, Foundation of potential theory, (Berlin, 1929)

[22] K.N. Pustovalov, P.M. Nagorskiy, Atmosphere and Ocean Optics, 29(8), 647-653 (2016)

[23] B.P. Demidovich, I.A. Maron, Osnovy vychislitel'noy matematiki [Basis if numerical mathematics], (Moscow, 1966) (in Russian)

[24] P.P. Firstov, R.R. Akbashev, R. Holzworth, N. V. Cherneva, and B. M. Shevtsov, Izvestiya, Atmospheric and Oceanic Physics, 53(1), 24-31 (2017) https://doi:10.1134/10.1134/S0001433817010066

[25] P.P. Firstov, R.R. Akbashev, N.A. Zharinov et al., Journal of Volcanology and Seismology, 13(3), 172-184 (2019) https://doi:10.1134/S0742046319030035 